\title{
The (F)utility of the thallium-201 quantitative lung/myocardial ratio in the detection of coronary artery disease
}

\author{
Richard L. Wahl, Bharath Kumar, Daniel R. Biello, and Tom R. Miller \\ The Edward Mallinckrodt Institute of Radiology, Washington University School of Medicine, 510 South Kingshighway, St. Louis, \\ MO 63110, USA
}

\begin{abstract}
Exercise-induced increases in pulmonary uptake of thallium-201 $\left({ }^{201} \mathrm{Tl}\right)$ have been associated with exerciseinduced myocardial dysfunction. To evaluate this phenomenon more replicably, a quantitative semi-automated computer program was used to generate, from anterior exercise and delayed views, lung-myocardial ratios (LMR) of ${ }^{201} \mathrm{Tl}$ uptake in 78 patients [ 40 normal, 38 with coronary artery disease (CAD)]. Patients with CAD had a significantly higher mean exercise lung myocardial ratio (EXLMR) than normals ( 30.8 vs. $27.3 ; P<0.003$ ). In patients with adequate exercise ( $\geq 85 \%$ of an age-adjusted maximal heart rate), the EXLMRs of CAD patients were significantly higher than those of normals ( 29.7 vs. $25.5 ; P=0.003$ ). However, this difference between $\mathrm{CAD}$ and normal patients was not apparent in a patient subgroup with submaximal exercise levels ( $<85 \%$ of an age-adjusted maximal heart rate). In both normal and CAD patients, EXLMR decreased with increasing exercise levels $(r=-0.555 ; P=0.007)$. In patients with ${ }^{201} \mathrm{Tl}$ scans lacking visually defined perfusion defects (visually normal), an elevated LMR detected $60 \%$ of CAD cases with $81 \%$ specificity. A considerably elevated EXLMR in patients achieving adequate exercise should suggest the presence of CAD, even if there are no visually apparent cardiac perfusion defects. With submaximal exercise, however, the EXLMR is not a useful discriminator between $\mathrm{CAD}$ patients and normals.
\end{abstract}

Key words: Thallium-201 - Coronary artery disease - Exercise testing

Increased lung uptake of thallium-201 ${ }^{201} \mathrm{Tl}$ in resting and exercise studies has been shown to be related to elevated left atrial pressure, increased severity of coronary artery disease (CAD), increased numbers of myocardial perfusion defects, and a worsened prognosis compared with individuals having normal lung ${ }^{201}$ Tl uptake (Bingham et al. 1980; Boucher et al. 1980; Gibson et al. 1982; Kushner et al. 1981; Lahiri et al. 1984; Tamaki et al. 1982). Studies demonstrating these relationships have been conducted by qualitative assessment or heavily operator-dependent quantitation of lung ${ }^{201} \mathrm{Tl}$ uptake or lung/heart ratios (Bingham et al. 1980; Boucher et al. 1980; Gibson et al. 1982;

Offprint requests to: Richard L. Wahl, M.D., Division of Nuclear Medicine, Department of Internal Medicine, Box 0028, The University of Michigan Medical Center, Ann Arbor, MI 48109, USA
Kushner et al. 1981; Lahiri et al. 1984; Tamaki et al. 1982). In this report, we evaluated lung uptake of ${ }^{201} \mathrm{Tl}$ by comparing it to myocardial uptake through the use of a new computer program allowing enhanced replicability of data. In this study, upright exercise was on a bicycle ergometer as opposed to prior studies in which exercise was performed either supine or upright on a treadmill. The relationship of the lung-myocardial ratio (LMR) to exercise level (heart rate), presence and severity of CAD, and the significance of the LMR in a patient subset whose ${ }^{201} \mathrm{Tl}$ scans lacked perfusion defects were evaluated using this methodology.

\section{Patients and methods}

Patients who underwent cardiac catheterization between May 1980 and August 1983 at the Edward Mallinckrodt Institute of Radiology and who had undergone exercise ${ }^{201} \mathrm{Tl}$ imaging were included (except for patients who had had previous coronary artery bypass surgery). There were 78 patients, 38 with $\mathrm{CAD}$ ( $\geq 50 \%$ stenosis of one or more major coronary artery or ergonovine-induced spasm) and 40 normals. There were 48 men and 30 women. The patients ranged in age from 27 to 69 years, with a mean age of 52.4 years. The mean age of patients with CAD was 56.6. Sixty percent of the patients with CAD and $59 \%$ of the normals were men.

\section{Thallium-201 stress imaging}

Patients underwent graded upright bicycle exercise in the fasting state using a modified Bruce protocol. The ECG, blood pressure and pulse rate were monitored throughout the study, with an attempt being made to raise the patient's heart rate to at least $85 \%$ of an age-adjusted maximal rate on an incremental schedule or until chest pain, dyspnea, leg fatigue, or significant ST segment depression occurred. At peak exercise, $1.5-2.1 \mathrm{mCi}^{201} \mathrm{Tl}$ was injected intravenously, followed by a saline flush. Exercise was continued for an additional $60 \mathrm{~s}$ following the injection, if possible.

Patient imaging began 3-10 min after injection in the $35^{\circ}$ left anterior oblique position; anterior and $70^{\circ} \mathrm{LAO}$ views followed sequentially. The anterior view was collected approximately $10-17 \mathrm{~min}$ post-injection. After a 3 - to $4-\mathrm{h}$ delay, the patient was reimaged in a similar fashion. A portable gamma camera (LEM) with a one-quarter inch crystal, fitted with a low-energy all-purpose collimator that was interfaced to a dedicated nuclear medicine computer 


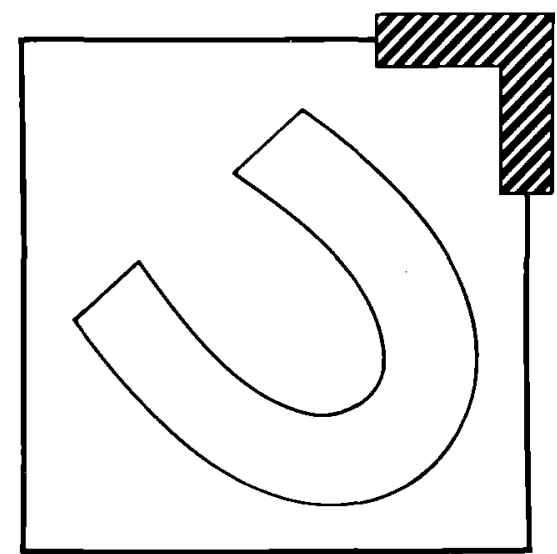

Fig. 1. The lung-myocardial ratio was determined from an anterior view at exercise (EXLMR) or on delayed views (DLMR) by drawing a rectangle about the heart on a computer system. The upper outer third of the perimeter of the box defined the lung to be evaluated for counts, while the maximal heart activity within the box was selected for the heart activity (see Patients and methods). This method has high replicability

system was used to collect $500,000-900,000$ counts/image over a 7- to 10 -min interval at the $80 \mathrm{keV}$ mercury photopeak. The digital images were displayed on a $64 \times 64$ matrix with 256 gray scale levels. In addition, standard transparency film images were obtained.

All studies were read by a panel of at least three physicians for perfusion defects and a consensus reading was determined. Image interpretation was aided by a computer display system, which allowed varying degrees of background subtraction. Perfusion defects that were fixed or which changed on delayed imaging were noted.

The quantitative LMR was determined on the anterior views obtained immediately post-exercise and on the delayed anterior images in all patients. A rectangle was drawn manually about the left ventricle on the display screen, with borders approximately 4-6 pixels outside the left ventricular myocardium. Following nine-point smoothing of the image, the average pixel value of the upper and outer one-third of this border (which lies in the left lung) was determined and divided by the maximum nine-point smoothed myocardial pixel value, which was automatically determined by the computer (Fig. 1). This ratio times 100 produced the LMRs used in the study. The LMR determined immediately post-exercise is called the EXLMR and the LMR on delayed images the DLMR. The replicability of this number was analyzed for inter- and intra-observer variability by having the same observer reprocess 11 patients' data several weeks after initial processing and also by having a second operator process these same patients.

All patients underwent coronary arteriography in standard fashion. Coronary arteriograms were interpreted by two physicians. Coronary artery disease was defined as a $50 \%$ (as compared to a proximal normal portion of the artery) or greater reduction in intra-luminal diameter of the right coronary artery, left main, left anterior descending or left circumflex coronary artery. The presence of ergonovine-induced spasm, which was assessed in several cases, was also considered positive for CAD.

Patient histories obtained by the physician conducting the exercise study, including the medications as well as ECG findings with exercise, and symptoms at exercise were reviewed.

A paired $t$-test and regression analysis were used to assess inter- and intra-observer variability in quantitative data processing. The entire population data was assessed by Student's $t$-test.

\section{Results}

The quantitative LMR was found to be reproducible with a low intra-operator variability $(r=0.95)$ and a low interoperator variability $(r=0.94)$.

The entire patient population was analyzed using the quantitative EXLMR. Patients with CAD had a significantly higher EXLMR $(30.8 \pm 5.2 \mathrm{SD} ; \pm 0.85 \mathrm{SEM})$ than patients with no evidence of significant $\overline{C A D}$ by cardiac catheterization $(27.3 \pm 5.9 \mathrm{SD} ; \pm 0.83 \mathrm{SEM} ; P<0.003)$. In addition, the EXLMR was positively but weakly correlated with the severity of CAD $(r=0.419, P<0.0005)$. The DLMRs were not, however, significantly different between the two groups.

In both patient groups the LMR increased from exercise to delay (with a $23 \%$ increase in normals, and a $15 \%$ increase in CAD; $P=$ NS). Thus the delayed LMR increased 5.5 LMR points in normals and 4.2 in CAD patients (compared to the EXLMR; $P=\mathrm{NS}$ ).

In both the CAD and normal groups the EXLMR was inversely related to exercise level $(r=-0.535 ; P=0.001)$. The CAD patient group had a slightly lower mean exercise level $(81.3 \%)$ than the normal group $(87.7 \%)$. When patients with exercise levels $<85 \%$ of the age-predicted maximum were evaluated separately from those with exercise levels $\geq 85 \%$ of the age-predicted maximum heart rate, the EXLMR was not correlated with the severity (as determined by the number of vessels with $\geq 50 \%$ stenosis) ( $r=$ $0.26, P=0.17)$ or the presence $(r=0.108, P=0.585)$ of CAD. This differed from the adequate $(>85 \%$ of predicted maximum heart rate) exercise group in which the EXLMR was correlated with the severity $(r=0.52, P=0.004)$ and presence of CAD $(r=0.42, P=0.003)$.

The mean EXLMR for CAD patients with adequate exercise $(n=21)$ was $29.7 \pm 4.6 \mathrm{SD}( \pm 1.02 \mathrm{SEM})$ and for normal patients with adequate exercise $(n=27)$ was $25.5 \pm 4.3 \mathrm{SD}( \pm 0.83 \mathrm{SEM} ; P=0.003$; Fig. 2$)$. Both were lower than the means EXLMRs for patients with inadequate exercise $(\mathrm{CAD}=32.2 \pm 5.6 \mathrm{SD}, \pm 1.36 \mathrm{SEM}, n=17$; normal $=30.8 \pm 7.5 \mathrm{SD}, \quad \pm 2.17 \mathrm{SEM}, \quad n=12) \quad(P=\mathrm{NS}$; Fig. 3).

Thirty-six patients in the group had ${ }^{201} \mathrm{Tl}$ scans lacking perfusion defects; of these, 10 had CAD (5 with one vessel and 5 with two vessel disease). If a quantitative LMR was determined to be greater than 28.3 (which was chosen as a dividing line between normal and $\mathrm{CAD}$, based on analysis of first 20 patients), then 7 of the 10 cases of CAD would have been detected ( $70 \%$ sensitivity). However, there would have been 7 false-positive diagnoses ( $73 \%$ specificity). If only patients who had adequate exercise levels are included in the evaluation, the sensitivity of the LMR test in this population drops slightly to $60 \%$ (3 out of 5), but the specificity rises to $81 \%$ (13 out of 16 ). If only patients with "inadequate exercise" $(<85 \%)$ are included, then sensitivity rises to $80 \%$ (4 out of 5 ), but specificity drops to $60 \%$ (6 out of 10$)$. 


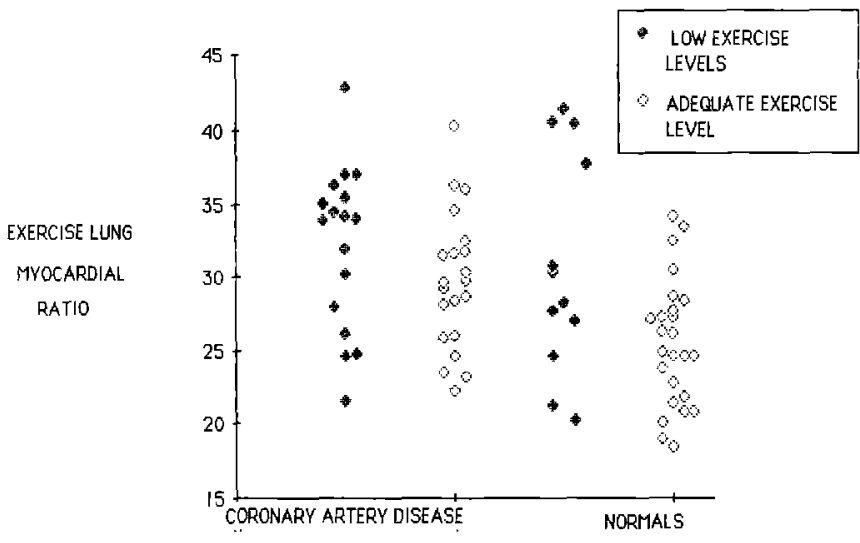

Fig. 2. Exercise lung-myocardial ratios (EXLMR) at "adequate exercise" (heart rate $\geq 85 \%$ of an age - adjusted maximum-predicted heart rate; open diamonds). Note highly significant difference between population means, but large overlap between patient groups $(\mathrm{CAD}$ mean $=29.7 \pm 1.02 \mathrm{SEM}$; normal mean $=25.5 \pm$ 0.83 SEM ; $P=0.003$ ). EXLMRs at "inadequate exercise" (heart rate $<85 \%$ of an age-adjusted maximum-predicted heart rate; closed diamonds). Note the lack of significant difference between the CAD and normal patients and total overlap of values (CAD mean $=32.2 \pm 1.36 \mathrm{SEM}$; normal mean $=30.8 \pm 2.17 \mathrm{SEM} ; \quad P=$ NS). CAD patients are shown in left two columns. Normals are shown in right two columns

\section{Discussion}

The semi-automated quantitative LMR that we have developed is somewhat similar to the qualitative or more operator-dependent quantitative methods of other investigators in this field (Boucher et al. 1980; Gibson et al. 1982; Kushner et al. 1981; Lahiri et al. 1984; Tamaki et al. 1982). The EXLMR is evevated in patients with CAD whose exercise raises their heart rate to an age-adjusted maximal value as well as in normal and CAD patients who exercise inadequately. Since the EXLMR can be performed in less than 1 min on our computer system, it does not add significantly to the time or cost of the study.

The LMR values reported here are lower than those reported elsewhere in the literature (Boucher et al. 1980; Kushner et al. 1981; Lahiri et al. 1984). This is likely due to two factors: 1 . The exercise in this study was upright (which has been shown to result in lower PA wedge pressure at peak exercise than supine exercise). 2. The denominator of the LMR, myocardial uptake, was computer-selected from a retively small area representing a weighted average of only 9 pixels of maximal activity (maximum myocardial pixel was 9 point-smoothed). The denominator is thus likely higher than that in other studies in which myocardial activity was flagged over a larger area by an observer as "the best wall" of the heart (Boucher et al. 1980; Kushner et al. 1981).

In contrast to some prior studies, a relatively strong relationship was seen between increased exercise and decreased LMR in both normal and CAD patient groups (Lahiri et al. 1984). In fact, in the patients who were "inadequately exercised" (exercise level $<85 \%$ of age-adjusted predicted maximum heart rate), the LMR was not able to separate CAD patients from normals. This finding differs from that of Tamaki et al. (1982), who showed differences between normal and CAD patients at rest; however, his patients generally had severe cardiac disease. By contrast, most of our patients were ambulatory outpatients referred for evaluation of somewhat atypical chest pain.

We also saw in both patients with CAD and in normals that exercise LMRs were lower than delayed LMRs - in contrast to other studies in which the EXLMRs of CAD patients were higher than those on delayed images (Boucher et al. 1980; Kushner et al. 1981). The magnitude of increase was similar for CAD and normal patients. The reason for this difference is uncertain, but may be related to our upright exercise protocol.

If multiple perfusion defects are present on ${ }^{201} \mathrm{Tl}$ scan, the patient is strongly suspected of having CAD, and the LMR adds only supporting data to the suspected diagnosis of CAD. There are, however, individuals in whom "relatively balanced" CAD may result in no focal perfusion defects on ${ }^{201} \mathrm{Tl}$ scan. In these persons, the lung uptake of ${ }^{201} \mathrm{Tl}$ relative to myocardial uptake may be the only scintigraphic manifestation of CAD and detectable only by elevation of the LMR (Boucher et al. 1980; Kushner et al. 1981).

Certainly an elevation in the LMR is not specific for CAD (Bingham et al. 1980; Lahiri et al. 1984; Tamaki et al. 1982) and probably can result from any type of myocardial dysfunction. As an example, one of our patients with a very high LMR had normal large coronary vessels and an apparently non-ischemic cardiomypathy.

The mechanism and tissue localization of increased lung ${ }^{201} \mathrm{Tl}$ is still unclear. A relationship between increased LA pressure, slower pulmonary transit time and increased ${ }^{201} \mathrm{Tl}$ uptake has been shown (Bingham et al. 1980). Certainly, with exercise-induced ischemia left atrial pressure can rise (Barry et al. 1974; Dwyer 1970). Recently pulmonary ${ }^{201} \mathrm{Tl}$ uptake has been shown to be related to extravascular pulmonary fluid volume, which may result from a variety of causes (Slutsky and Higgins 1984). The contribution of increased ${ }^{201} \mathrm{Tl}$ uptake in the lungs and decreased ${ }^{201} \mathrm{Tl}$ uptake in the heart has not been totally addressed. Clearly increased ${ }^{201} \mathrm{Tl}$ in the lungs occurs, but ${ }^{201} \mathrm{Tl}$ uptake in the heart certainly could be relatively decreased in severe CAD. The LMR ratio should mathematically enhance these processes related to CAD.

In conclusion, the EXLMR is capable of separating groups of patients with $\mathrm{CAD}$ from normals when exercise is "adequate". There is, however, significant overlap between individual normal and CAD patients when exercise is adequate. The EXLMR in our hands was incapable of separating CAD from normal patients when exercise was not adequate. A significant elevation of the EXLMR in a patient with a ${ }^{201} \mathrm{Tl}$ scan devoid of perfusion defects who has achieved "adequate exercise" should raise the suspicion of CAD. Quantitation of the LMR is recommended when exercise ${ }^{201} \mathrm{Tl}$ scans lack perfusion defects and may improve sensitivity of planar imaging for the detection of $C A D$, but it must be used with caution.

\section{References}

Atkins HL, Budinger TF, Lebowitz E, Ansari AN, Green MW, Fairchild RG, Ellis KJ (1977) Thallium-201 for medical use. III. Distribution and physical imaging properties. J Nucl Med 18:133-140

Barry WH, Brooker JZ, Alderman EL, Harrison DC (1974) Changes in diastolic stiffness and tone of the left ventricle during angina pectoris. Circulation 49:255

Bingham JB, McKusick KA, Strauss HW, Boucher CA, Pohost 
GM, Godley FA (1980) Influence of coronary artery disease on pulmonary uptake of thallium-201. Am $J$ Cardiol $46: 821-826$

Boucher CA, Zir LM, Beller GA, Okada RD, McKusick KA, Strauss HW, Pohost GM (1980) Increased lung uptake of thallium-201 during exercise myocardial imaging : clinical, hemodynamic and angiographic implications in patients with coronary artery disease. Am J Cardiol 46:189-196

Dwyer EM (1970) Left ventricular presure volume alterations and regional disorders of contraction during myocardial ischemia induced by atrial pacing. Circulation $42: 1111$

Gibson RS, Watson DD, Carabello BA, Holt ND, Beller GA (1982) Clinical implications of increased lung uptake of thallium-201 during exercise scintigraphy 2 weeks after myocardial infarction. Am J Cardiol 49:1586-1593

Kushner FG, Okada RD, Kirshenbaum HD, Boucher CA, Strauss
HW, Prohost GM (1981) Lung thallium-201 uptake after stress testing in patients with coronary artery disease. Circulation $63: 341-347$

Lahiri A, O'Hara MJ, Bowles MJ, Crawley JCW, Raftery EB (1984) Influence of left ventricular function and severity of coronary artery disease on exercise-induced pulmonary thallium201 uptake. Int J Cardiol 5:475-490

Slutsky RA, Higgins CB (1984) Thallium scintigraphy in experimental toxic pulmonary edema: relationship to extravascular pulmonary fluid. J Nucl Med 25:581-591

Tamaki N, Itoh H, Ishii Y, Yonekura Y, Yamamoto K, Torizuka K, Konishi Y, Hikasa Y, Kambara H, Kawai C (1982) Hemodynamic significance of increased lung uptake of thallium-201. AJR 138:223-228

Received June 20, 1985/November 9, 1985 